\title{
A Multifaceted Quality Improvement Initiative to Reduce Unnecessary Laboratory Testing on Internal Medicine Inpatient Wards
}

Inka Toman, MD, MSc; Pamela Mathura, MBA, and Narmin Kassam, MD, MHPE

\section{About the Authors}

Inka Toman is an Assistant Clinical Professor in the Department of Medicine at the University of Alberta. She practices General Internal Medicine at the University of Alberta Hospital and the Sturgeon Community Hospital in Edmonton, Alberta, Canada.

Pamela Mathura, MBA is a Clinical Lecturer in the Department of Medicine at the University of Alberta. She works as a senior quality consultant for Alberta Health Services in Edmonton, Alberta, Canada.

Narmin Kassam, MD, MHPE is a Professor and Associate Chair in the Department of Medicine at the University of Alberta. She is the Deputy Clinical Department Head of Medicine for Alberta Health Services, Edmonton Zone. She practices General Internal Medicine at the University of Alberta Hospital in Edmonton, Alberta, Canada.

Correspondence: Narmin Kassam nkassam@ualberta.ca

Submitted: March 3, 2018. Accepted: May 27, 2019. Published: May 1, 2019. DOI: 10.22374/cjgim.v15i2.357

\section{Abstract \\ Background}

The American and Canadian Choosing Wisely campaigns recommend against routine complete blood count $(\mathrm{CBC})$ and chemistry testing in the face of clinical stability in the inpatient internal medicine setting.

\section{Problem}

Patients on internal medicine units commonly have daily lab tests ordered at admission and lab testing is often bundled. Four 'core' lab tests (CBC, electrolytes, creatinine, and urea) account for more than half of all lab tests performed.

\section{Methods}

The Model for Improvement and the Donabedian framework was used to define the problem, evaluate the baseline state, and generate targeted improvements. A quality improvement (QI) initiative consisting of education and process change was implemented on one general internal medicine unit and multiple plan-do-study-act cycles were carried out. The outcome measure was the total number of core labs performed, and the process measure was the proportion of patients with tests ordered on a repeating daily basis.

\section{Results}

The initiative led to an $18.9 \%$ decrease in the total number of core labs ordered and an $18.2 \%$ absolute decrease in repeating daily lab orders.

\section{Conclusions}

A multifaceted QI initiative aimed at reducing unnecessary lab testing was successful at reducing the number of lab tests ordered and changing lab ordering process. 


\section{Resume \\ Contexte}

Les campagnes américaine et canadienne "Choisir judicieusement" recommandent de ne pas effectuer de tests de routine de numération globulaire complète $(\mathrm{CBC})$ et de chimie face à la stabilité clinique des patients hospitalisés en médecine interne.

\section{Problème}

Les patients des unités de médecine interne doivent généralement passer des examens de laboratoire quotidiens à l'admission et les analyses de laboratoire sont souvent regroupées. Quatre tests de laboratoire "de base" (NFS, électrolytes, créatinine et urée) représentent plus de la moitié de tous les tests de laboratoire effectués.

\section{Méthodes}

Le modèle d'amélioration et le cadre donabédien ont été utilisés pour définir le problème, évaluer létat de référence et générer des améliorations ciblées. Une initiative d’amélioration de la qualité (AQ) consistant en un changement d'éducation et de processus a été mise en œuvre dans une unité de médecine interne générale et plusieurs cycles "planifier-faire-étudier-agir" ont été réalisés. La mesure des résultats était le nombre total de laboratoires de base effectués, et la mesure des processus était la proportion de patients dont les tests commandés se répétaient quotidiennement.

\section{Résultats}

Cette initiative a permis de réduire de 18,9\% le nombre total de laboratoires de base commandés et de $18,2 \%$ en valeur absolue le nombre de commandes quotidiennes répétitives.

\section{Conclusions}

Une initiative d'AQ à multiples facettes visant à réduire les tests de laboratoire inutiles a permis de réduire le nombre de tests de laboratoire commandés et de modifier le processus de commande des laboratoires.

Diagnostic testing is a significant driver of rising health care costs. ${ }^{1}$ Overuse of lab tests in various clinical settings has been estimated at $20.6 \%{ }^{2}$ The Canadian Society of Internal Medicine and the Society of Hospital Medicine Choosing Wisely campaigns recommend against routine complete blood count (CBC) and chemistry testing in clinically stable medical inpatients. ${ }^{3,4}$

Overutilization and unnecessary lab testing negatively impact patient experience by contributing to iatrogenic anemia and discomfort. ${ }^{5}$ Volume of phlebotomy is independently associated with the development of iatrogenic anemia. ${ }^{6}$ Hospital-acquired anemia is associated with increased length of stay and hospital readmission rates. ${ }^{7}$ Furthermore, phlebotomy is painful and causes anxiety.

Research shows that residents are more likely to order unnecessary lab tests than hospitalist physicians. ${ }^{8}$ Educating residents about the harms and costs of unnecessary lab testing to reduce excess lab ordering has been unsuccessful used in isolation. ${ }^{9}$ Multiple research groups have implemented multifaceted QI interventions that have proven successful at reducing unnecessary lab testing without adverse safety consequences. ${ }^{10-12}$ However, previous groups did not use the Model for Improvement and Donabedian framework, Prosci ADKAR model or Lewin change management theory to create sustainable changes and transform institution culture.

The objective of this project performed on an inpatient medical unit at an academic Canadian hospital was to determine baseline rates of unnecessary lab testing, to build an understanding of this problem, and to develop and implement a QI intervention to decrease unnecessary lab testing on general internal medicine (GIM) wards. Institution culture was identified as a major cause of unnecessary lab testing, and through the use of validated change management strategies, our goal was to change to current culture at our site. A multidisciplinary project team was established to promote stakeholder engagement, change acceptance and sustainability. The QI initiative was multifaceted, consisting of education and process change, and developed through iterative 
plan-do-study-act (PDSA) cycles. Our a priori expectation was that the QI intervention would decrease the total number of $\mathrm{CBC}$, electrolytes, creatinine and urea tests (hereafter referred to as 'core labs') done during a one-year study period by $10 \%$ to reduce patient harm and system costs.

\section{Methods \\ Study Setting}

This project was conducted at a 650-bed teaching and research hospital in Canada. The hospital has five adult GIM units with 18 beds each, and annual admission of nearly 4000 GIM patients per year. Unit 5D2 is a clinical teaching unit with an attending physician, residents and medical students who rotate every week, four weeks and eight weeks, respectively. The physician healthcare team is cohorted on one unit. Our institution uses a combined system of paper charting and an electronic health record (EHR). Patient care orders and progress notes are written in the paper chart, and test results are displayed in the EHR. GIM uses a preprinted admission order set. On this order form CBC, electrolytes, creatinine, urea and prothrombin time are prewritten and ordered by marking a checkbox. $\mathrm{CBC}$ and prothrombin time can be selected alone, but electrolytes, creatinine, and urea are bundled. The clinician has a blank space to write an ordering frequency of their choice (e.g., daily). Other lab tests must be written in by hand.

\section{Quality Framework}

The Model for Improvement framework was used. Key components were: baseline process review by a frontline team (defining the opportunity), QI tool completion and local process data review (building the understanding), focused aim determination, and iterative PDSA cycles. ${ }^{13}$

\section{Quality Team}

The multidisciplinary team included a GIM resident, attending physician, laboratory services representative, unit clerk, registered nurse, unit manager and QI consultant.

\section{Baseline Lab Data}

Laboratory services provided data sorted by test type and location. Six months of lab tests from 2016 were used for Pareto analysis, which displays frequency along with a cumulative percentage to determine which lab tests were ordered most frequently on GIM units.

\section{Chart Audit}

A spot chart audit was performed by one reviewer on the study unit. Patient charts were eligible for inclusion if they were direct admissions from the emergency department and had a length of stay less than or equal to 10 days. Admission orders were reviewed to determine if lab tests were ordered as the daily frequency at admission, which tests were ordered, and the duration of daily lab tests. Subsequent orders were reviewed to determine if daily lab orders were changed or stopped. Progress notes were reviewed for comments that addressed lab testing.

\section{Resident Survey}

An online survey inquiring about lab ordering practices, specifically targeting questions around unnecessary lab testing, was emailed to all residents in the Internal Medicine program. A minimum response rate of $10 \%$ of eligible residents was selected. Response to the survey was voluntary and anonymous.

\section{Process Mapping}

An in-person process mapping session was organized to generate the cross-functional (swim lane) process map. This session transparently identified the current process of lab orders completed by the frontline multidisciplinary team.

\section{Cause-Effect Analysis}

An Ishikawa diagram outlining the causes of unnecessary lab ordering was developed through brainstorming by the QI team following the chart audit, resident survey, and process mapping session.

\section{Plan-Do-Study-Act Cycles}

A multifaceted intervention was initiated in January 2017. Discussions with the QI team occurred every four to eight weeks and changes were made accordingly. Four iterative PDSA cycles occurred from January to December 2017, as originally described by Shewhart. ${ }^{14}$

\section{Measures}

The Donabedian model of structure, process and outcome was used for evaluation. ${ }^{15}$ The outcome measure selected was the volume of the four most frequently ordered lab tests - CBC, electrolytes, creatinine, and urea (core labs) - done on unit 5D2. Laboratory Services provided a database with all lab tests done on internal medicine units from January 2016 to February 2018. The process measure was the percentage of patients on the study unit who had core labs ordered on a repeating daily basis and was available from February to December 2017. This was obtained from the lab Kardex by dividing the total number of admitted patients by the number of patients with lab tests ordered as daily. Balance measures included length of stay, readmission rates, and 30-day mortality. 


\section{Analysis}

The outcome measure and process measure were graphically analyzed using a run chart. ${ }^{16}$ Cost savings were calculated by volume reduction of core labs one-year postintervention compared to one-year preintervention based on reference median test costs at six Canadian diagnostic sites.

\section{Ethics and Reporting}

The ARECCI ethics screening tool was used to determine the level of ethical review required for this project. ${ }^{17}$ The ARECCI score was one, such that the project involves minimal risk and no ethics board review was required. The SQUIRE 2.0 statement was used to develop this manuscript. ${ }^{18}$

\section{Results}

\section{Baseline Lab Data Analysis}

Pareto analysis of six months of lab data from 2016 identified the four most frequently ordered lab tests on GIM wards: CBC, electrolytes, creatinine, and urea (core labs). As a proportion of all lab tests done on GIM wards, CBC comprised 19\%, electrolytes $14 \%$, creatinine $13 \%$, urea $11 \%$, prothrombin time $4 \%$ and all other lab tests $39 \%$.

\section{Chart Audit}

Thirteen charts met inclusion criteria for the spot chart audit. In 9/13 (69\%) charts, lab tests were ordered "daily" at admission. Four of nine were ordered daily indefinitely, 4/9 were ordered as "daily x 3 " and 1/9 was ordered as "daily x 2 ". Daily lab testing was always bundled. Nine of nine (100\%) daily lab test orders included all core labs. On review of the progress notes, only one note was identified that explicitly addressed daily lab testing.

\section{Resident Survey}

Thirty-eight of 101 (38\%) internal medicine residents responded to the survey. Ninety-seven percent of residents order CBC daily at admission in greater than $75 \%$ of patients. Upon transfer of admitted patients to the wards, $68 \%$ of residents always review lab orders. Fewer than $30 \%$ of residents always review lab orders on daily rounding. With regards to unnecessary lab ordering, $95 \%$ of residents admit to ordering unnecessary lab tests at least once per week and $92 \%$ feel inappropriate lab testing is a problem at our institution. When asked why residents order unnecessary lab tests, the most commonly cited reason was institution culture. Other reasons included a lack of ordering guidelines, concern about missing something, the design of the preprinted admission order set and fear of receiving a negative performance review.

\section{Process Mapping}

The detailed steps of provider and patient roles in lab test ordering are outlined in the cross-functional map (Figure 1). An important finding from this mapping session was that physicians are often unaware which lab tests have been ordered until the test results are displayed in the EHR, but the unit clerk keeps a record of all pending patient lab tests and records this in the lab Kardex, a word document kept on the unit computer and updated daily. Through this mapping session, it was apparent that the lab Kardex was not available to physicians, despite the useful information it contained, and many physicians were unaware of its existence.

\section{Cause-Effect Analysis}

Many factors leading to unnecessary lab testing were identified, which stemmed from the patient, the process, residents and the equipment and forms used (diagram not shown). Important root causes were: labs were frequently ordered daily at admission, lab order frequency was not formally reassessed on rounds, residents were unaware of upcoming lab tests, residents lacked education about the harms of unnecessary lab testing, communication gaps existed within the patient care team, and preprinted admission order sets drive recurrent ordering of bundled tests.

\section{QI Intervention Development and Implementation}

A multifaceted intervention consisting of education and process changes was developed and implemented on unit 5D2 in January 2017 and is summarized in Table 1. Monthly education was provided to students, residents and attending physicians by an email, a poster displayed on the unit, and an in-person presentation. Education of the harms of unnecessary lab testing and the process changes occurring on the unit was provided. Multiple process changes were implemented during the intervention. The research team met regularly to discuss the successes and shortcomings of the interventions, and changes were made as required.

The first PDSA cycle involved education and a daily printout of the lab Kardex to be reviewed on physician rounds. The lab Kardex was put directly onto the doctor's clipboard for daily review. The second PDSA cycle involved weekly follow-up on the unit by a member of the QI team to provide ongoing education. In the third PDSA cycle, daily orders on the lab Kardex were highlighted. The fourth PDSA cycle added an auto-substitution label that was placed onto the order sheet by the unit clerk when labs were ordered on a recurring daily basis and were substituted for "daily $\mathrm{x} 3$ days" when signed by a physician. Formal education ended in July, but the use of the lab Kardex, highlighting daily orders and the auto-substitution label is ongoing. 


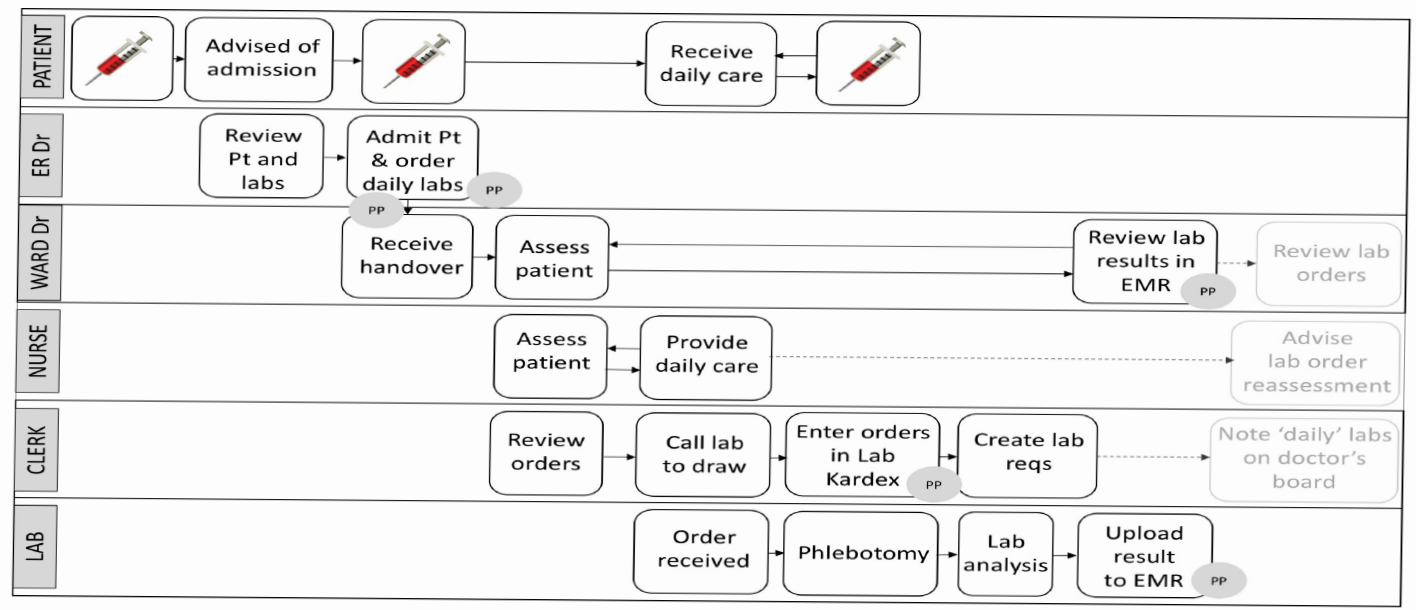

Figure 1. Cross-functional (swim lane) process map generated by a multidisciplinary team. Each provider and patient role in the process of lab ordering was mapped. Areas identified for improvement are marked as pain-points. Each role is displayed in a swim lane, process steps that always occur are displayed as solid arrows and steps that only sometimes occur are displayed as dashed arrows. $\mathrm{PP}=$ pain-point; syringe $=$ patient phlebotomy; $\mathrm{Pt}=$ Patient; $\mathrm{Dr}=$ Doctor; $\mathrm{EMR}=$ electronic medical record; req $=$ requisition.

Table 1. Multifaceted quality improvement intervention on study unit 5D2 during 2017. Monthly education involved an email to all medical staff, a unit poster and an in-person presentation. The lab Kardex lists all patients on the unit and the lab tests that have been ordered for each patient. Weekly follow-up was done early in the intervention. In March lab tests ordered as daily were highlighted on the lab Kardex for closer review by the medical team. After June an auto-substitution label was pasted onto the order sheet by the unit clerk when labs were ordered as daily. This replaced "daily" orders with "daily x 3 days" once signed by a physician.

\begin{tabular}{|l|l|}
\hline \multicolumn{1}{|c|}{ Date (2017) } & \multicolumn{1}{c|}{ Intervention } \\
\hline January - June & Monthly education \\
\hline January - ongoing & Lab Kardex printed daily \\
\hline February - April & Weekly follow up on unit \\
\hline March - ongoing & Highlight daily orders \\
\hline June - ongoing & Auto-substitution label \\
\hline
\end{tabular}

\section{Measures}

The volume of core labs performed on unit 5D2 during the study period decreased following the quality improvement intervention. A run chart of the outcome measure shows a downward shift following the intervention (Figure 2). The median volume of core labs every four weeks preintervention was 2768.5 , compared to 2245.5 postintervention, which represents an $18.9 \%$ decrease. Each of the four core labs measured individually displayed the same trend towards decreased ordering over time (data not shown). The percentage of patients on the unit that had lab tests ordered as daily showed a significant reduction from the beginning of the intervention to the implementation of the last PDSA cycle. Initially, $23.7 \%$ of patients had daily lab orders but following the intervention a median of $5.5 \%$ of patients had daily lab orders, which represents an absolute decrease of $18.2 \%$. (Figure 3). Balance measures were not available for unit $5 \mathrm{D} 2$, but no adverse effects were seen at a division or institution level with respect to the length of stay, readmission rates and 30-day mortality (data not shown). Neither the staff on the unit nor Laboratory Services noted any unintended consequences.

\section{Cost Savings}

The decreased total volume of core labs on unit 5D2 in the year following the intervention represents cost savings of \$15,049 CAD.

\section{Limitations}

The outcome and process measures were based on change over time, which assumes that the study unit remains constant. In reality, a GIM unit is in constant flux, with patient turnover occurring daily, medical illnesses varying seasonally, and medical 


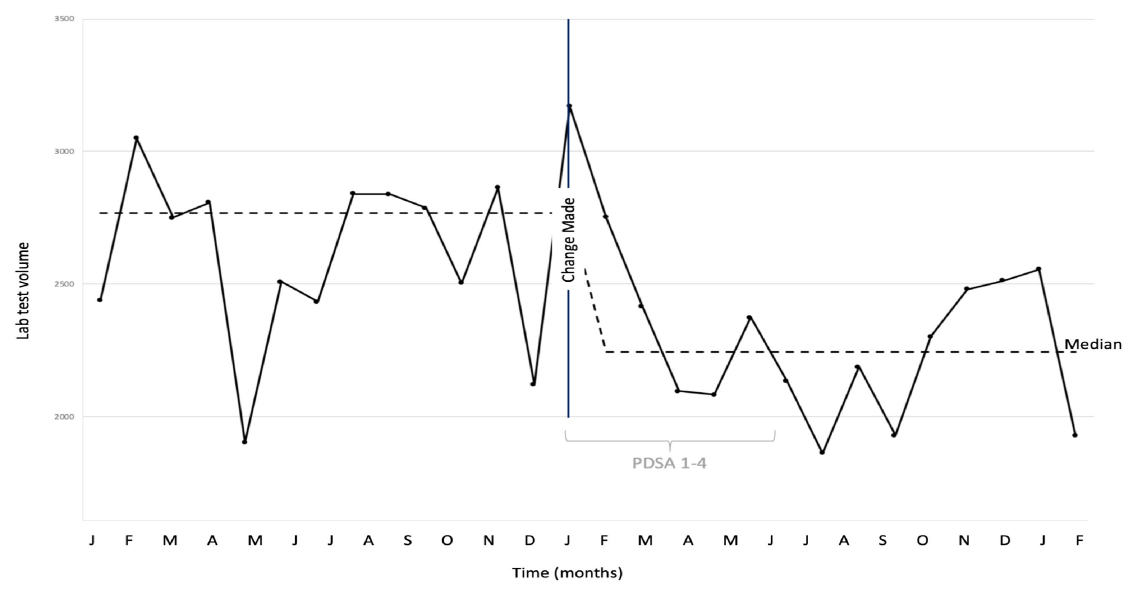

Figure 2. Run chart of the outcome measure pre- and postintervention. The total number of core labs (CBC, electrolytes, creatinine, and urea) performed on the study unit from January 2016 to February 2018 is shown. The quality improvement intervention started in January 2017 and the last PDSA cycle started in June 2017. The median volume of lab tests done every four weeks preintervention was 2768.5 tests compared to 2245.5 following the start of the intervention, which represents a $18.9 \%$ decrease.

staff rotating weekly. To mitigate the variable nature of the GIM unit we studied our measures for a one-year preintervention and more than one-year postintervention to allow variation to regress to the mean over time so that variability should not confound our observations.

The aim of this project was to decrease unnecessary lab testing, but there are no validated tools to determine the necessity of a lab test; furthermore, a very labour-intensive chart review would be required, and necessity is challenging to assess retrospectively. There is extensive literature confirming that unnecessary lab testing is a ubiquitous problem in internal medicine units. ${ }^{3,4} \mathrm{~A}$ survey of residents at our institution demonstrated that unnecessary lab testing is a problem based on their experiences, and a chart review showed a variety of lab ordering practices that lacked clinical relevance. Based on this evidence we determined that unnecessary lab testing was an issue at our institution, and we assumed that a decrease in the total volume of lab tests performed without any adverse consequences was representative of fewer unnecessary tests.

This quality improvement intervention was carried out on a clinical teaching unit staffed by residents and medical students. Evidence shows that resident physicians order more tests than attending physicians. ${ }^{8}$ The generalizability of our intervention to a non-teaching unit, therefore, has limitations and may require modification for spread to other units and other hospitals. Evaluation of the current state and stakeholder engagement will be required for future project spread and sustainability and is currently underway.

\section{Discussion}

A multifaceted quality improvement intervention consisting of education and process change was developed with the goal of building awareness about unnecessary lab ordering and changing the culture at our institution. In order to create stakeholder engagement, we created a multidisciplinary QI team to jointly develop the quality improvement intervention. A reduction in the volume of core lab testing by $10 \%$ over a one-year study period on GIM unit 5D2 was targeted. With successive PDSA cycles, the total number of core labs done on the study unit decreased by $18.9 \%$.

To create a sustainable change, the process of lab ordering needed modification. By educating physicians and making the lab Kardex available for daily patient rounds, we were able to decrease the occurrence of future recurring labs, or daily orders. 


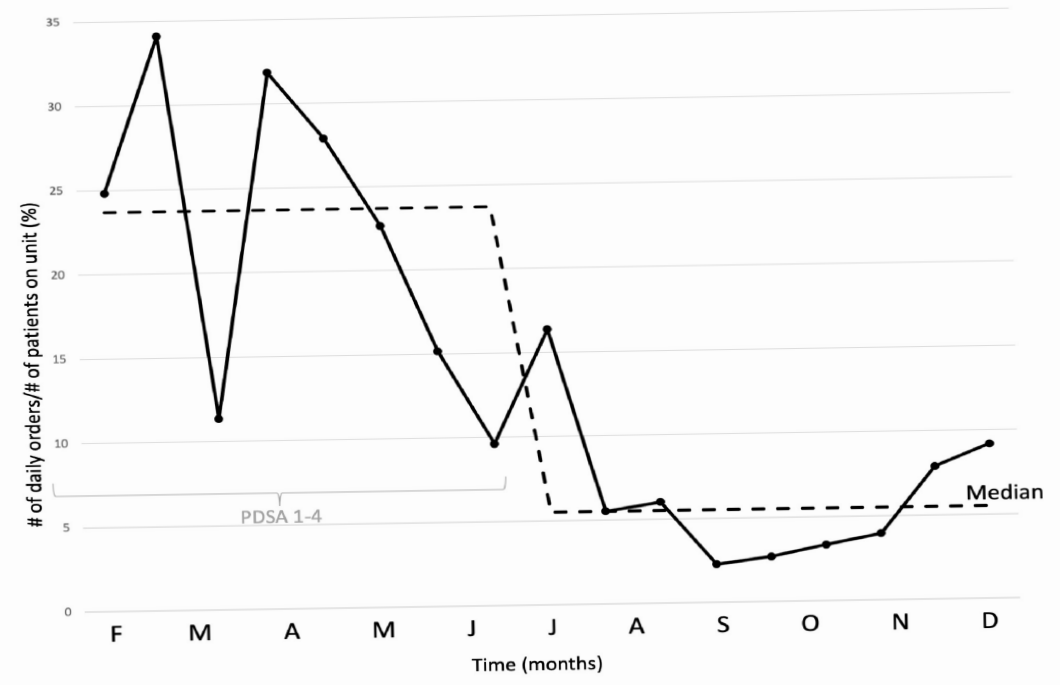

Figure 3. Run chart of the process measure postintervention. The percentage of patients on the unit with daily lab test orders from February 2017 to December 2017 is shown. The quality improvement intervention started in January 2017 and the last PDSA cycle started in June 2017. At the beginning of the intervention, $23.7 \%$ of patients were receiving daily lab tests, compared to $5.5 \%$ following the final PDSA cycle.

With the reduction in lab testing following our intervention, we observed annual cost savings on unit 5D2 of \$15,049 CAD. This represents projected annual cost savings on all five GIM units of $\$ 86,725$ CAD annually with the spread of this project and does not account for other lab tests which may have followed a similar decline. There were no unintended adverse outcomes. Despite formal education ending in July 2017, the unit staff has continued to educate incoming students and residents about this QI initiative on unit 5D2 and use of the lab Kardex. This has led to sustainable decreases in the outcome and process measures which are observed many months following the intervention.

The Institute for Healthcare Improvement (IHI) uses the Model for Improvement framework to implement and spread sustainable changes in the healthcare setting, ${ }^{13}$ which we applied to this project. Creating a multidisciplinary team and building an understanding of the problem at our institution were critical early steps for the successful implementation of this initiative. Multidisciplinary process mapping identified a communication gap between clinicians and the unit clerk, and a bridge for this gap - the lab Kardex. In fact, the unit clerk realized this document was already in existence and could easily be printed and placed on the doctor's clipboard for daily patient rounds. In doing this, we created stakeholder engagement, change acceptance and a sustainable process change. Pareto analysis of lab data identified the most frequently ordered lab tests on GIM units. As expected, these are the same tests that can be selected on the preprinted admission order set. Therefore, the volume of these tests done on unit 5D2 was selected as our outcome measure. A chart audit and resident survey demonstrated that labs are frequently ordered as future recurring events; thus, the ordering labs on a recurring daily basis was used as our process measure.

Various quality improvement tools allowed us to define our system's current state and identify areas for improvement. A major issue was that institution culture is a driving force behind unnecessary lab ordering. Changing institution culture is very challenging, so a deliberate sequence of quality improvement interventions was created in iterative PDSA cycles based on the principles of the Prosci ADKAR model for change and the Lewin change model to support permanent change management. ${ }^{19,20}$ The first iteration of our intervention consisted of monthly education to build awareness and create a desire for change. The lab Kardex provided clinicians with the knowledge to change and 
encouraged them to trust their abilities as physicians to perform mindful lab ordering. In subsequent PDSA cycles weekly follow-up, highlighting daily orders and an auto-substitution label was used to promote further knowledge, ability, and reinforcement of the change. These initial PDSA cycles provided perspective to the healthcare team about the routines and culture that surrounded lab ordering practices at our institution previously and presented new methods to support their abilities as healthcare providers. Since then we have made changes to the admission order set that will reinforce the change and support sustainability. The new admission order set unbundles all lab tests, removes the urea test as a preprinted option and allows the physician to select from various finite frequencies. This form will be used by all GIM units, selected subspecialty units, and the family medicine service. Daily printing of the lab Kardex has been spread to all five GIM units.

By 2020, our institution will be transitioning to a complete EHR for patient care orders and for test results. Based on the present study, and the work of previous groups,${ }^{21}$ bundlings of lab orders and future recurring tests within the EHR will be limited.

\section{Conclusions}

Following the Model for Improvement and the Donabedian model provided a systematic approach for the frontline multidisciplinary team to define the opportunity and build process understanding. The initiative led to an $18.9 \%$ decrease in the total number of core labs ordered and a significant decrease in repeating daily lab orders. Change interventions supported by measurement to determine change impact and stakeholder engagement were generated. This framework provided the foundation for change acceptance, sustainment and QI project success. With this multifaceted quality improvement initiative, we have created a sustainable intervention to reduce unnecessary lab testing, with plans to spread to multiple units at our institution and beyond. This has the potential to reduce patient harm and create cost savings.

\section{REFERENCES}

1. Stuebing EA, Miner TJ. Surgical vampires and rising health care expenditure: Reducing the cost of daily phlebotomy. Arch Surg. 2011;146:524-527.

2. Zhi M, Ding EL, Theisen-Toupal J, Whelan J, Arnaout R. The landscape of inappropriate laboratory testing: A 15-year meta-analysis. PLoS One. 2013;8:e78962.
3. Society of Hospital Medicine. Five things physicians and patients should question. http://www.choosingwisely.org/societies/society-of-hospitalmedicine-adult/. Published 2013.

4. Canadian Society of Internal Medicine. Five Things Physicians and Patients Should Question.; 2018. https://choosingwiselycanada.org/internal-medicine/.

5. Thavendiranathan P, Bagai A, Ebidia A, Detsky AS, Choudhry NK. Do blood tests cause anemia in hospitalized patients? The effect of diagnostic phlebotomy on hemoglobin and hematocrit levels. J Gen Intern Med. 2005;20:520-524.

6. Salisbury AC, Reid KJ, Alexander KP, et al. Diagnostic blood loss from phlebotomy and hospital-acquired anemia during acute myocardial infarction. Arch Intern Med. 2011;146:524-527.

7. Lin RJ, Evans AT, Chused AE, Unterbrink ME. Anemia in general medical inpatients prolongs length of stay and increases 30-day unplanned readmission rate. South Med J. 2013;106:316-320.

8. Ellenbogen MI, Ma M, Christensen NP, Lee J, O'Leary KJ. Differences in Routine Laboratory Ordering Between a Teaching Service and a Hospitalist Service at a Single Academic Medical Center. South Med J. 2017;110:25-30.

9. Melendez-Rosado J, Thompson KM, Cowdell JC, et al. Reducing unnecessary testing: An intervention to improve resident ordering practices. Postgrad Med J. 2017;93:476-479.

10. Attali M, Barel Y, Somin M, et al. A cost-effective method for reducing the volume of laboratory tests in a university-associated teaching hospital. $\mathrm{Mt}$ Sinai J Med. 2006;73:787-794.

11. Corson AH, Fan VS, White T, et al. A multifaceted hospitalist quality improvement intervention: Decreased frequency of common labs. J Hosp Med. 2015;10:390-395.

12. Yarbrough PM, Kukhareva P V., Horton D, Edholm K, Kawamoto K. Multifaceted intervention including education, rounding checklist implementation, cost feedback, and financial incentives reduces inpatient laboratory costs. J Hosp Med. 2016;11:348-354.

13. Langley G, Moen RD, Nolan KM, Nolan TW, Norman CL, Provost LP. The Improvement Guide: A Practical Approach to Enhancing Organizational Performance (2nd Edition).; 2009.

14. Best M, Neuhauser D. Walter A Shewhart, 1924, and the Hawthorne factory. Qual Saf Heal Care. 2006;15:142-143.

15. Donabedian A. The quality of medical care. Science (80- ). 1978;200:856-864.

16. Perla RJ, Provost LP, Murray SK. The run chart: a simple analytical tool for learning from variation in healthcare processes. BMJ Qual Saf. 2011;20:46-51.

17. Hagen B, O’Beirne M, Desai S, Stingl M, Pachnowski CA, Hayward S. Innovations in the Ethical Review of Health-Related Quality Improvement and Research: The Alberta Research Ethics Community Consensus Initiative (ARECCI). Healthc Policy. 2007;2:e164-77.

18. Ogrinc G, Davies L, Goodman D, Batalden P, Davidoff F, Stevens D. SQUIRE 2.0 ( Standards for QUality Improvement Reporting Excellence ). Am J Med Qual. 2015;25:986-992.

19. Boca GD. Adkar Model Vs . Quality Management Change. In: International Conference "Risk in Contemporary Economy."; 2013:246-563.

20. Lewin K. Frontiers in Group Dynamics: Concept, Method and Reality in Social Science; Social Equilibria and Social Change. Hum Relations. 1947;1:5-41.

21. May TA, Clancy M, Critchfield J, et al. Reducing unnecessary inpatient laboratory testing in a teaching hospital. Am J Clin Pathol. 2006;126:200-206. 